\title{
Ilizarov method in treatment of neurological foot deformities
}

\author{
A. Kirienko ${ }^{1}$, E. Malagoli ${ }^{1}$, G. Lucchesi ${ }^{2}$ \\ ${ }^{1}$ Humanitas Clinical and Research Center - IRCCS, Rozzano, Milan, Italy \\ ${ }^{2}$ Orthopaedic Clinic, Department of Surgery and Translational Medicine, University of Florence, Florence, Italy
}

\begin{abstract}
Introduction Diseases related to the lesions of upper or lower motor neurons, such as spina bifida, myopathy, and residual polio, often cause severe deformities of the foot and ankle. The treatment goal is to convert a deformed and rigid foot into a plantigrade foot. Matherial and method We treated 51 neurological feet from 2001 to 2018 with the Ilizarov method. They were polio outcomes in 27 cases, 17 feet were affected by spina bifida, 6 other patients had Charcot-Marie-Tooth disease. Results Results were assessed by comparing appearance and function before and after surgery described by Dimeglio, and modified by Dong Y.L. et al. Before surgery, there were 33 moderate and 28 severe deformities (average preoperative scores, 6.3). The mean time required to correct a deformity was 37.1 days (range, 22-58 days) and the mean time for stabilization was 67.2 days (range, 45-98 days). At latest follow-up (mean 7.42 years, from 13 month to 16 years), clinical outcomes and patient satisfaction were considered acceptable. 28 patients achieved an excellent outcome and 21 patients had a good outcome, two feet were classified as having a poor result. Nearly $86 \%$ of patients (44 patients) were satisfied with their outcomes and replied they would repeat their procedure. Complications include infection in $15 \%$ of K-wire tracts, Dysesthesia in 5 cases, superficial necrosis in 3. One patient developed knee contracture. 2 patients were re-operated with V osteotomy. Conclusions The Ilizarov method represents a great resource in the treatment of severe neurological deformities of the foot.
\end{abstract}

Keywords: neurologic foot, complex foot deformity, Ilizarov method, osteotomy, arthrodesis, arthroereisis, distraction, Charcot-Marie-Tooth disease

\section{INTRODUCTION}

Diseases related to the lesions of upper or lower motor neurons, such as cerebral paralysis, spina bifida, myopathy, and residual polio, often cause severe deformities of the foot and ankle [1-3]. Treatment of a neurological foot with this severe deformity is difficult, and deformity recurrence is a common problem [4]. The treatment goal for a rigid deformity of the neurological foot is to convert a deformed and rigid foot into a plantigrade foot [2, 4]. Interventions on soft tissues combined with osteotomies are the preferred treatment. However, the complexity of these deformities with stiffness or instability often require more radical procedures such as triple primary arthrodesis or talectomy in difficult cases [5, 6-8]. Potential disadvantages of these disabling procedures include reducing foot height, lowering the malleoli, and high rates of complications, which suggest the need to find other options and alternative treatment.

The Ilizarov method and its application to the foot and ankle have greatly expanded over the past two decades. It has become an alternative to traditional methods with soft tissue interventions combined with osteotomies to treat rigid neurological foot deformities or [2, 9-12]. Patients with complex deformities, previous multiple operations, with soft tissue compromise or deep ulcerations in the foot are the categories most likely to benefit from the use of the Ilizarov techniques $[4,13,14]$. This work aims to assess the potential of the Ilizarov method in the treatment of neurological foot deformity to correct complex equino-cavus-varus deformities and other types of rigid deformities, improve patient functions, and to achieve patient satisfaction. We also sought to provide an algorithmic approach to treating neurological foot deformities based on our experience. It depends on the type of pathology, the type of deformity or instability of the foot.

We used various techniques of interventions with the method of Ilizarov. We have described the techniques of:

1. Corrections of rigid or residual deformities with corrective osteotomies and distraction:

a) V- or Y-osteotomies;

b) Other combined osteotomies of hindfoot (Dwyer type), midfoot (cuboid-navicular, cuboid-cuneiform).

2. Arthroereisis - surgery to limit pathological or uncontrolled movement of foot joints.

3. Stabilization of foot joints with triple arthrodesis with the Ilizarov fixator and tendon transfer.

\section{MATERIAL AND METHOD}

We have treated 51 neurological feet from 2001 to 2018 with the Ilizarov method. They were polio outcomes in 27 cases, with different foot deformities: 12 with equinus-varus, 5 with equinus-valgus, 2 with equinus-varus- adductus, 5 with equinuscavus-varus, 3 feet with talus and valgus. Six of these patients were treated with "closed method" without bone osteotomy; $\mathrm{V}$-shaped osteotomy was performed in 5 cases; 2 cases we have treated with supramalleolar osteotomy, 10 cases with triple arthrodesis and 3 cases with ankle arthrodesis, arthrodesis of Chopart in 1 case. Additional procedures were: 13 cases of limb

[d Kirienko A., Malagoli E., Lucchesi G. Ilizarov method in treatment of neurological foot deformities. Genij Ortopedii, 2021, vol. 27, no 3, pp. 383-389. DOI 10.18019/1028-4427-2021-27-3-383-389 
lengthening, 8 cases of Achilles tendon lengthening, and one knee arthrodesis.

17 feet were affected by spina bifida with complex foot deformities in talus in 13 feet ( 5 with vertical heel and 8 with valgus), 4 with equinus-varus foot, 4 with ankle deformity, knee contraction in flexion in 4 cases. We have performed proximal osteotomy of the tibia (6 cases), supramalleolar osteotomy (5 cases), subatalar and Chopart arthrodesis (8), anterior arthrorises (3), V-shaped osteotomy (1), Y-shaped osteotomy (4), toe correction (10). Six other patients had Charcot-Marie-Tooth disease with equino-cavus-varus foot. We have treated 2 cases with Y-osteotomy and other 4 patients with triple arthrodesis and progressive distraction with application of Ilizarov. Results were assessed by comparing appearance and function before and after surgery [15]. Dimeglio et al. [16] described a detailed scoring system for classification of congenital clubfoot based on measurement of four parameters: (1) equinus deviation in the sagittal plane; (2) varus deviation in the frontal plane; (3) derotation of the calcaneopedal block in the horizontal plane; and (4) adduction of the forefoot relative to the hind foot in the horizontal plane. This system has been reported to be the most reliable for classification of the severity of equinocavovarus deformity [17]. In the current study, we used the modified by Dong Y.L. et al. [18] classification of Dimeglio et al. They modified the original 4-point scale and used a 2-point scale for each parameter. Instead of additional points in the original system of Dimeglio et al. (points for the presence of medial creases, a posterior crease, cavus, and poor calf musculature [7]), we measured a fifth parameter (cavus of the forefoot relative to the hind foot in the sagittal plane) in a 2-point scale. Total scores ranged from 0 to 10 points and were allocated to four grades: severe, 7 to 10 points; moderate, 4 to 6 points; mild, 1 to 3 points; and postural, 0 points. Morphologic and functional outcomes were determined using the criteria reported by Kling et al. [19]. Excellent was defined as a plantigrade foot with no fixed or postural deformities or callosities, allowing wearing of normal shoes. Good was defined as a residual deformity less than $5^{\circ}$ varus, valgus, or equinus deformity. There were no callosities and normal shoes could be worn. Recurrent equinocavovarus deformity or an overcorrection greater than $5^{\circ}$ was considered a poor outcome. Patients' overall satisfaction also was investigated. Patient's (or parent's in some cases) satisfaction was determined by questionnaire that asked them whether they were satisfied with outcomes and whether they would undergo the operation again if they had the same preoperative deformities. Patients were considered satisfied when they replied they were satisfied and they would undergo the operation again. Ethical approval was obtained from the Hospital's Ethics Committee.

\section{SURGICAL TECHNIQUES}

\section{Corrective osteotomies and distraction}

Indications for open treatment are rigid deformities in the adolescent and the adult when the tibiotalar joint is abnormal and does not allow normal gliding of the bony surfaces. The V-osteotomy is indicated whenever lengthening and correction are required simultaneously; the Y-osteotomy is exclusively corrective.

\section{Method with V-osteotomy}

The V-osteotomy is the result of a combination of the oblique osteotomy of the posterior calcaneus and the anterior calcaneal-talar osteotomy. The two osteotomy cuts intersect at an acute angle of 60-70 degrees at the plantar tip on the lower surface of the calcaneus. We use a $1.8-\mathrm{mm} \mathrm{K}$-wire and position it on the lateral side of the foot at the level of the proposed osteotomy to help determine the position of the incision site under fluoroscopic guidance. The peroneal tendons and the sural nerve are identified, and the superficial peroneal nerve and extensor digitorum brevis are designed. V-osteotomy should be done by percutaneous technique with a straight osteotome from two small lateral incisions under fluoroscopic control during surgery. First approach to the calcaneus is achieved by a vertical incision about $2.5 \mathrm{~cm}$ just posterior to the lateral malleolus. The direction of skin incision corresponds to the direction of future distraction with the aim to prevent traction of the suture during correction. We perform the osteotomy with a Lambotte osteotome, $20 \mathrm{~mm}$ or $15 \mathrm{~mm}$ in width. After insertion of the osteotome and arriving at the bone periosteum, the osteotome is rotated in the direction of the line of osteotomy under fluoroscopic control. If necessary, the Achilles tendon is lengthened by percutaneous Z-shaped tendon lengthening to facilitate repositioning of the calcaneus. Another longitudinal incision of $2.5 \mathrm{~cm}$ just anterior of the apex of the lateral malleolus will be done for the anterior branch of osteotomy in the projection of the anterior part of the subtalar joint. The direction of skin incision is longitudinal corresponding to the direction of future distraction, with the aim to prevent traction of the suture during correction. Initially going deeper with periosteal elevator and after with the osteotome. The rotation of the osteotome is performed after attaining bone surface. In this way, it reduces risk of damage to the superficial peroneal nerve. Under fluoroscopic guidance, once again we are using the positioning of the K-wire as a reference, to guide our osteotomy. During osteotomy of the neck of the talus and anterior part of calcaneus, the dorsal and plantar structures were protected with elevators. The Ilizarov frame consists of two full proximal tibial rings, one hind food and one forefoot half ring. Fixation of the proximal full ring is accomplished using 1.8-mm olive wires. The foot half rings are fixed using olive wires in the calcaneus (three wires) and the mid-forefoot (three wires). Two smooth talar wires are inserted from lateral to medial and attached to the tibial ring without tension by threaded rods with female posts and a wire fixator. An optional olive wire, cut right before the olive (called by the author a "rein wire"), is inserted on the proximal fragment of the calcaneus then attached to the tibial ring to prevent diastasis of the subtalar joint. The optional second and third "rein olive wire" can be inserted in the anterior plantar fragment of the calcaneus to prevent diastasis of calcaneo-cuboid joint during distraction and on the anterior fragment of the talus to prevent distraction of talar-navicular joint. Pinning of the toes is necessary to prevent the formation of claw-toe deformity. Correction is started three-five days postoperatively. Follow-up visits are conducted every ten to fourteen days during distraction and on a monthly basis during consolidation.

The appropriate rods between the leg support and the half rings should be lengthened $1-1.5 \mathrm{~mm}$ per day and the 
rods between the leg support and the metatarsal half ring should be compressed $1-1.5 \mathrm{~mm}$ per day. The goal is to distract $1 \mathrm{~mm} /$ day on the osteotomy site in order to create the space needed for corrective movement of the bony segments and to prevent premature consolidation

After one week, radiographs are taken to observe how the distraction is progressing and to look for subluxations of the tibiotalar joint. It is good practice to correct adduction and supination of the forefoot when there is already bone regeneration present in the region of the calcaneal-talar osteotomy, which is elastic enough to tolerate rotation and medial distraction. In order to eliminate the equinocavus deformity, the forefoot is rotated into dorsiflexion, pivoting the forefoot and midfoot on the calcaneal-talar osteotomy., Residual varus deformity of the calcaneus after the acute correction may be eliminated by lengthening the medial threaded rod on the calcaneal half-ring. The period of fixation lasts for approximately 3 months. The apparatus is removed as soon as consolidation occurs, then progressive weight-bearing with crutches is initiated.

\section{Method with Y-osteotomy}

Two incisions are made at the osteotomy level similar to V-osteotomy. At first, the calcaneus is osteotomized (the oblique posterior branch of the "Y"). Secondly, the vertical osteotomy of the calcaneus (the vertical branch of the "Y"), and finally the calcaneal-talar osteotomy (anterior branch of the "Y") is completed. The resulting shape is a three-ray star with rays equally spaced 120 degrees apart. The assembly of the device is the same as that used for the V-osteotomy. It is not necessary to insert a wire to prevent calcaneal-cuboid diastasis. The hinges are positioned on the medial and lateral threaded rods of the calcaneal half-ring, at the peak of the medial malleolus and then slightly anterior, about $0.5 \mathrm{~cm}$ with respect to the anatomical axis of the tibia. Equinus is corrected by lowering the calcaneus and raising the forefoot with respect to the talar body. The talus is fixed to the leg support with two crossed wires. The calcaneal half-ring, rotating around the axis of the hinges by the push forces of the posterior rod, shifts the calcaneus distally and anteriorly in a rotational movement greater than that in the $\mathrm{V}$ osteotomy. The eventual necessity to further lower the calcaneus for the correction of equinus is performed by equally lengthening the three rods on the calcaneal half-ring, whereas varus deformity is corrected by lengthening only the medial rod.

The forefoot is simultaneously subjected to proximal traction, in the opposite direction of the equinus and the cavus deformities (dorsal flexion). Supination and equinus was corrected by differentiated shortening of the two vertical threaded rods between the leg support and the metatarsal half-ring (lateral rod faster than medial rod). As in previous V-osteotomy, if necessary, Achilles tendon is lengthened by percutaneous $\mathrm{Z}$-shaped tendon lengthening to facilitate repositioning of the calcaneus. To facilitate cavus deformity, it may be necessary to perform plantar fasciotomy. Correction is achieved through the movement of the bony fragments of the osteotomy in a relatively limited space. The major movements of the fragments are for the correction of the calcaneal and talar equinus. Since the deformity correction does not require lengthening, the cavus deformity does not require rapid correction. Only the posterior rod is lengthened
$2 \mathrm{~mm}$ every 24 hours for correction of equinus, while the plantar rods are lengthened $1 \mathrm{~mm}$ every 24 hours. After seven to eight days, a radiograph will show the progress of the distraction. The corrective program is continued based on skin disturbances until cavus and equinus are both resolved. Afterwards, attention is turned towards the elimination of the adduction deformity, which is achieved by lengthening the medial plantar rod between the calcaneal and metatarsal half-rings. The Y-osteotomy causes minimal skeletal lengthening, and therefore, offers three advantages, less bone regeneration is produced, so consolidation is faster; skin alteration is more easily contained and prevention of calcaneal-cuboid diastasis is unnecessary.

Combined osteotomies on the hind foot and mid-foot

Dwyer-Type Osteotomy of calcaneus (Dwyer type, midfoot osteotomy: cuboid-navicular, cuboid-cuneiform) are used on the hind foot and forefoot: Dwyer type osteotomy in the calcaneus and cuboid-navicular or cuboid-cuneiform were used. The assembly of the apparatus is similar to the one for $\mathrm{V}$-osteotomy. Correction is produced by wedge opening at the plantar base of the osteotomies. There is elongation of the foot skeleton with inevitable tension of the plantar soft tissue. To prevent danger of premature consolidation, the rein wires should be used in the fragment of the bone near osteotomies. The action of the apparatus on osteotomies must lead to a diastasis of $1 \mathrm{~mm}$ at their plantar end, but this objective may be hindered by trophic complications. As a result, the speed of distraction is a compromise between the need to diastase the osteotomic surfaces and that of limiting the extent of complications. It is better to overcorrect just a little bit to prevent recurrence in neurologic foot. Once the corrective result is obtained, the apparatus is left in fixation for 45-60 days and plaster cast for 30 days with gradual loading with crutches.

\section{Arthroereisis}

For severe paralytic talus foot, the method of the anterior arthroereisis is described (Fig. 1).

Arthroereisis is a technique used to limit the range of motion in a joint. In the foot, it is used to block abnormal dorsiflexion [20-22]. It is indicated for the treatment of paralytic calcaneal foot or the effects of hyperlengthening of the Achilles tendon after equinus correction. Although it is an uncommon procedure, it is useful for creating a stable and plantigrade foot in some patients. Arthroereisis is used as an alternative to arthrodesis, which is a more drastic procedure.

If Y-osteotomy is used for the correction of a talus foot with vertical calcaneus, the anterior arthroereisis can be obtained at the same time (Fig. 2). This method is indicated for paralytic talus foot or the outcomes of clubfoot in which there coexists hyper-extension of the Achilles tendon that allows this deformity. After performing the osteotomy and mounting the apparatus the foot remains fixed in maximum talus position. In this attitude, you get contact between the anterior edge of the tibial epiphysis and the talus neck. By staging the anterior branch of the Y-shape for corrective purposes, the contact remains unchanged and forms the bone obstacle to the dorsal extension of the foot (Fig. 1, d).

Clinical example of this technique is demonstrated in Figure 2. 


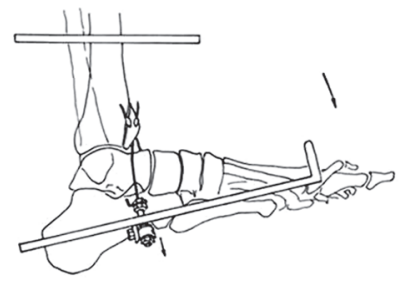

a

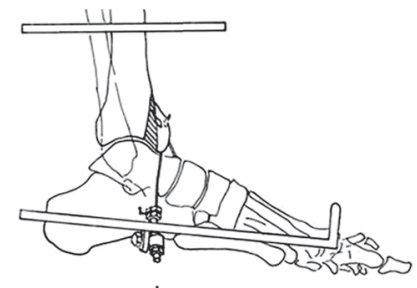

b

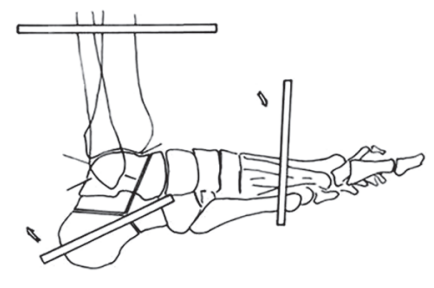

C

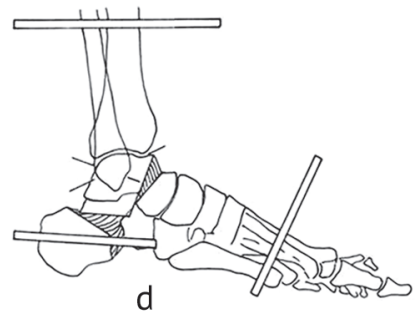

d

Fig. 1 Arthroereisis for limitation of dorsiflexion: $(\boldsymbol{a})$ arthroereisis with osteotomy of the tibial epiphysis and the fragment of the osteotomy is anchored with two olive wires that are distracted by grooved threaded rods; $(\boldsymbol{b})$ the traction of the fragment may also be achieved by bringing the foot to right $\left(90^{\circ}\right)$ or light equinus angles. The fragment must be guided to contact with the neck of the talus. After bone regeneration, a permanent block of ankle movement is created. This block corrects the abnormal dorsiflexion and results in a stable plantigrade foot; (c) diagram of mounting the apparatus on talus foot and attested in maximum dorsiflexion; Y-osteotomy. Note the contact between the anterior edge of the tibial epiphysis and the talus neck (TT). It is pointed out that the body of the talus is stuck with two crossed wires; $(\boldsymbol{d})$ after correction of deformity, there remains the contact in the TT point that makes the anterior arthroereisis

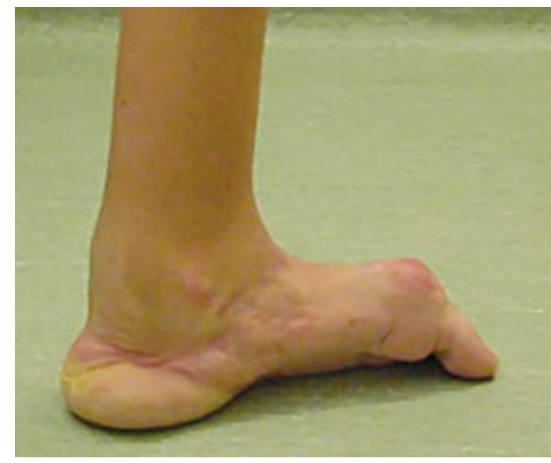

a

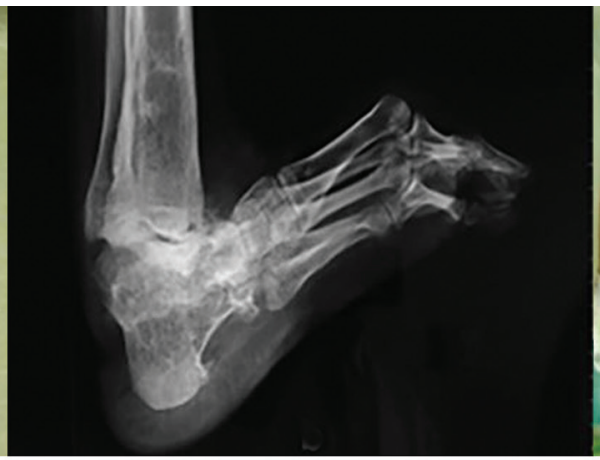

b

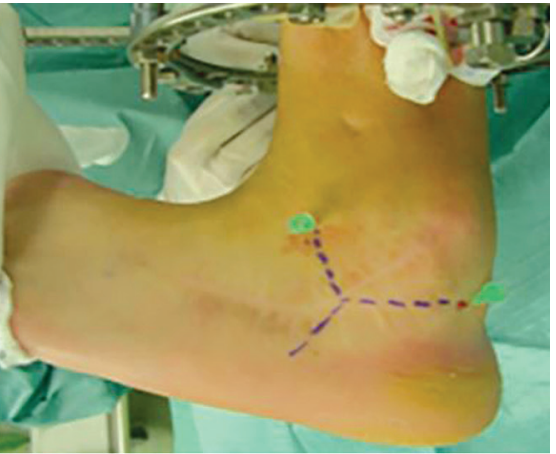

C

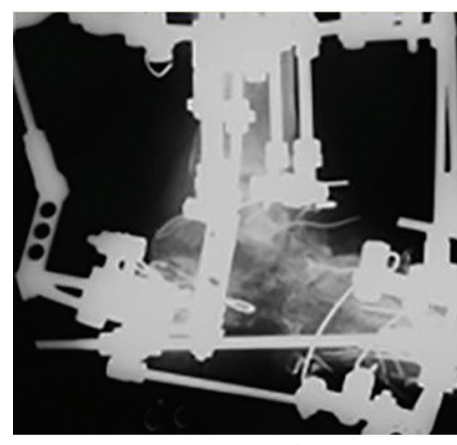

d

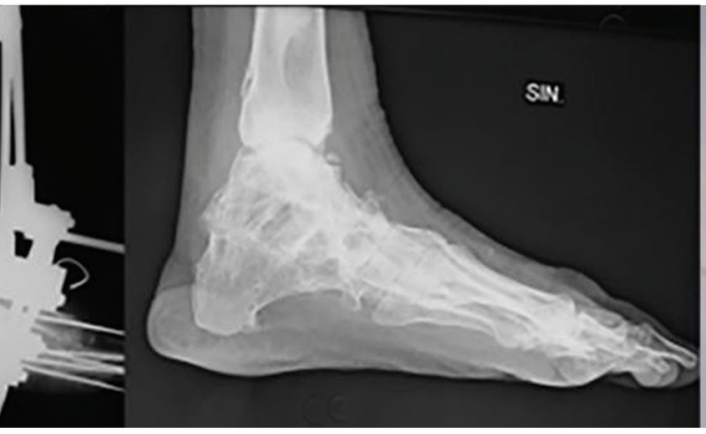

e

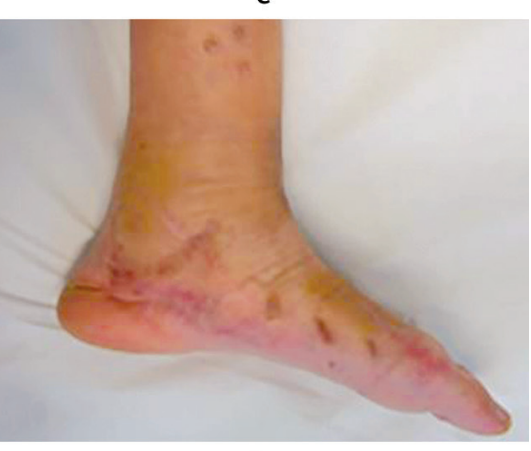

f

Fig. 2 42-year old male with spina bifida, vertical calnaneus: (a) clinical aspect of the deformity and contracture of the extensors; (b) X-ray of the deformity before treatment; $(c)$ intraoperative photo before performing Y-osteotomy and introducing cross wires into the talar body with dorsiflexion foot; $(\boldsymbol{d})$ X-ray check at the end of correction; $(\boldsymbol{e})$ X-ray 6 months after frame removal; $(\boldsymbol{f})$ clinical photo after treatment

\section{Stabilization or arthrodesis of the foot joints with} Ilizarov

Indications, differencies and advantages over traditional arthrodesis

The Ilizarov method is different and has advantages over traditional arthrodesis because may produce regenerated bone between the bony surfaces with gradual compression after the cartilage is removed and good bone contact is achieved. Moreover, it is possible to correct deformities, address loss of articular substance, and correct axial deviations of the foot without having to resort to bone resection, thereby preserving bone stock. Furthermore, deformity correction of the lower limb, such as lengthening, correction of axial deviations, can be combined with arthrodesis.

\section{Triple artrodesis}

The leg support is as previously described. Two crossed wires are introduced into the talus, and three opposing olive wires are introduced into the calcaneus. The skin should be pulled away from the subtalar joint and held tight during wire introduction. A wire is inserted into the cuboid and another into the navicular, running parallel to the sole of the foot; the skin should be pulled toward the forefoot. Two opposing olive wires are introduced into the metatarsals. A horseshoe-shaped ring is placed between the wires in such a way that the talar and navicular wires are on the cranial side and the calcaneal and cuboid wires are on the plantar side while the metatarsal wires are randomly fixed onto the straight plates. Compression is exerted on the subtalar joint by means of arching the talar wires down to the plantar ring and arching the calcaneal wires up to the plantar ring. Similarly, compression is exerted on the talarnavicular articulation and on the calcaneal-cuboid articulation by fixing and tightening the wires inserted in the navicular and the cuboid into more posterior holes of the horseshoeshaped ring. The metatarsal wires are used to stabilize the forefoot. The horseshoeshaped ring is rigidly fixed to the leg support. In case if the residual deformity remains, which cannot be corrected during surgery, a fixer is applied with a separate fixation of the forefoot and hindfoot and then a progressive correction with the corrective rods and junctions (Fig. 3). 


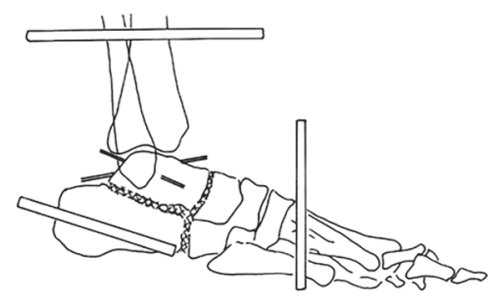

a

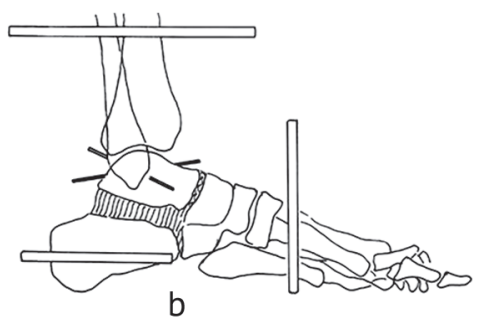

b
Fig. 3 (a) Triple arthrodesis of the foot and separate fixation of the hind foot and the forefoot with two half-rings; (b) progressive correction of equinus and varus of the calcaneus to obtain plantigrade foot by formation of a bone regerate in the seat of arthrodesis

\section{RESULTS}

The Ilizarov method combined with soft tissue release and/or osteotomy enabled good correction of rigid neurologic equino-cavo-varus deformities in all feet. Before surgery, there were 33 moderate and 28 severe deformities (average preoperative scores, 6.3), according to the modified system of Dimeglio et al. All were converted to postural feet or very mild foot deformities (average shortterm postoperative scores, 0.4 ) by distraction histiogenesis with soft tissue release and osteotomy or arthrodesis. The mean time required to correct a deformity was 37.1 days (range, 22-58 days) and the mean time for stabilization in the apparatus was 67.2 days (range, 45-98 days). Additional procedures were required to eliminate neuromuscular imbalance in 15 patients (12 feet).

In this series, postoperative complications occurred in six patients (six feet) including two pin-site infections, one bullous skin lesion, two tarsal tunnel syndrome, and one anterior impingement syndrome of the ankle. Recurrence of deformity requiring surgical correction occurred in four feet of three patients younger than 15 years. One patient (two feet) required reapplication of the Ilizarov apparatus, and the remaining two patients (two feet) required corrective osteotomy or soft tissue surgery at an average of 42.8 months (range, 29-53 months) after initial Ilizarov application. Mild recurrent or residual deformity was treated nonoperatively in six patients, higher prevalence in young patients with spina bifida. At latest follow-up at a mean of 7.42 years (range, 13 month to 16 years), clinical outcomes and patient satisfaction were considered acceptable. A postural plantigrade foot with no fixed or postural deformity was obtained in 24 feet. According to clinical outcome assessments, 28 patients achieved excellent outcomes and 21 patients had good outcomes. Two feet needing surgical correction for recurrent deformity were classified as having a poor result despite a final postural plantigrade appearance. Mean ankle ROM improved from $27^{\circ}$ preoperatively to $33.5^{\circ}$ postoperatively. Nearly $86 \%$ of patients (44 patients) were satisfied with their outcomes and replied they would repeat their procedure if they had the same preoperative deformities

Complications include infection in $15 \%$ of K-wire tracts, which were treated with oral antibiotics. Dysesthesia in 5 cases, superficial necrosis in 3 . One patient who underwent triple arthrodesis and lengthening developed knee contracture but refused further knee correction. Two patients with triple arthrodesis had recurrence and they were re-operated with V-osteotomy.

\section{DISCUSSION}

Treatment of rigid foot deformities in equino-cavusvarus or talus foot caused by upper and lower motor neuron injuries is often tricky, and recurrence is common. We reviewed our cases and described the Ilizarov technique to correct complex rigid foot deformities caused by neurological disorders. Although our study results suggest using the Ilizarov method, which may be a good option, the results must be interpreted considering the limitations of our study. This study was retrospective, and the patient group had several confounding variables: etiologies of deformity, the severity of the deformity, and age of the patients. A minimum follow-up of 13 months may be insufficient to evaluate clinical outcomes of correction, even though mean of follow-up have been more than seven years.

Generally, the Ilizarov procedure is chosen to treat complex foot deformities with rigid hindfoot deformity and a severe cavus component and maintain or increase foot length in a deformity correction process [2, 9-14]. It has advantages if compared with other demolition procedures because it is less invasive and allows the simultaneous correction of all components of a deformity without bone resection and shortening of the foot. Also, soft tissue vitality and function can be assessed directly, and the amount of correction can be adjusted accordingly [4]. In case of acute correction combined with talectomy, the talus must be removed entirely, and sometimes all or part of the navicular bone should also be removed to obtain the necessary correction [2, 8]. Although it has been reported $[8,23]$, the ability to walk after talectomy may be preserved, but minimal foot movement at the tibiotalar and mid-tarsal pseudarthrosis, foot height was low, and foot length was short.

Based on our experience in this study, we suggest an algorithmic approach to the treatment of complex neurological deformities of the feet using the Ilizarov method.

First, the hind foot flexibility is checked. If the hindfoot is not flexible and the varus heel deformity cannot be completely corrected, a lateral calcaneal osteotomy is performed. If the hindfoot deformity is completely correctable or corrected with this osteotomy, examine another component of the foot deformity: pes cavus. In some patients with mild cavus, soft tissue release including partial capsulotomy may be sufficient. If the cavus deformity is very rigid and severe, midtarsal osteotomy together with plantar fasciotomy and abductor tenotomy of the big toe is necessary. In this situation, gradual correction with Ilizarov can be used to correct severe deformities, midfoot with described osteotomies, 
and equinus correction is carried out in a closed manner. In our experience, the neurological equino-cavus-varus foot can be corrected without a dorsal closure wedge in the mid-tarsal osteotomy. For correction of the forefoot with severe supination deformity, a wedge-shaped osteotomy of plantar opening brings benefit to the complete correction of forefoot, as reported by others [24]. Subsequently, if the cavus foot remains, a dorsal wedge closure osteotomy of the first metatarsal base can be combined.

After osteotomy and soft tissue release, gradual correction using the Ilizarov method provides a foot with a more significant lengthening effect than a mid-tarsal osteotomy with dorsal wedge closure.

In terms of technical issues related to the Ilizarov procedure, the use of a half-pin or two crossed wires to fix the talus has helped restore the talus-calcaneal angle in cases with misaligned subtalar and mid-tarsal misalignment, as reported in other papers [4]. Another concept is that the deformed foot achieves a regular aspect with fixation of the talus and reduces the navicular on the head of the talus [25-28]. After reducing the dislocation of the talonavicular joint, a stable position should be maintained to enable osteogenesis in the distraction of the plantar-medial aspect of the cuneiform. This sequence of corrections is useful because wedge osteotomy without fixing the navicular bone can cause medial displacement of the scaphoid on the head of the talus, which can aggravate varus deformity of the hindfoot. [29].

When residual forefoot adduction remains, a wedgeclosing cuboid osteotomy is added successively.

In specific cases, triple arthrodesis or tendon transfers can be used after correction of the deformity in addition to this algorithmic approach to prevent a recurrence.

All major relapses of deformities requiring further surgical correction (mean range, 42.8 months) occurred in patients younger than 14 years with low motor neuron involvement (spinal dysraphism). Therefore, immobilization with braces or plaster cast at night is most important in younger patients who have not reached skeletal maturity yet. To reduce the risk of recurrence, it is essential to fully overcorrect all components of the deformity. Sometimes overcorrection of a deformity during the period of external fixation is helpful. Finally, and most importantly, the elimination of neuromuscular imbalance is essential to prevent relapse by combining the Ilizarov with arthrodesis with or without tendon transfer, especially in patients with low motor neuron diseases. We think that triple arthrodesis by Ilizarov method is not considered an aggressive surgery to correct neurological foot deformities. However, we could minimize bone resection to maintain foot height and length and reduce complication rates compared with primary triple arthrodesis.

Patients had several complications, including K-wire tract infection and dysesthesia, reported by other authors when the Ilizarov external fixator was used to correct foot deformities [30-33]. Generally, complications were minor and were managed non-surgically. Incidence and prognosis of perioperative complications were similar to those of previous publications on the Ilizarov correction of recurrent clubfoot deformity [31, 32]. However, in the case of anterior impingement syndrome, which can be considered a degenerative change, we resected the talus osteophyte.

Soft tissue distraction with or without foot bone callotasis with the Ilizarov method allows for a greater degree of correction of neurological rigid foot deformities, further minimizing surgery and scar formation. However, to reduce recurrence risk, it is essential to completely overcorrect all components of the deformity and eliminate neuromuscular imbalance by combining arthrodesis with fasciotomies and tendon transfer.

The Ilizarov method represents a great resource in the treatment of severe neurological deformities of the foot because it allows recovery of the plantigrade shape of the foot without further shortening and even allows its lengthening.

\section{REFERENCES}

1. Krause F.G., Wing K.J., Younger A.S. Neuromuscular issues in cavovarus foot. Foot Ankle Clin., 2008, vol. 13, no. 2, pp. 243-258. DOI: 10.1016/j. fcl.2008.02.003

2. Kucukkaya M., Kabukcuoglu Y., Kuzgun U. Management of the neuromuscular foot deformities with the Ilizarov method. Foot Ankle Int., 2002, vol. 23, no. 2, pp. 135-141. DOI: 10.1177/107110070202300210

3. McCluskey W.P., Lovell W.W., Cummings R.J. The cavovarus foot deformity: Etiology and management. Clin Orthop Relat Res., 1989, No 247, pp. 27-37.

4. Choi I.H., Yang M.S., Chung C.Y., Cho T.J., Sohn Y.J. The treatment of recurrent arthrogrypotic club foot in children by the Ilizarov method. A preliminary report. J. Bone Joint Surg. Br. 2001, vol. 83, no. 5, pp. 731-737. DOI: 10.1302/0301-620x.83b5.11019.

5. Green A.D., Fixsen J.A., Lloyd-Roberts G.C. Talectomy for arthrogryposis multiplex congenita. J. Bone Joint Surg. Br. 1984. Vol. 66, no. 5, pp. 697699. DOI: 10.1302/0301-620X.66B5.6389557

6. Pell R.F., Myerson M.S., Schon L.C. Clinical outcome after primary triple arthrodesis. J. Bone Joint Surg. Am. 2000 , vol. 82, no. 1, pp. 47-57. DOI: 10.2106/00004623-200001000-00006

7. Saltzman C.L., Fehrle M.J., Cooper R.R., Spencer E.C., Ponseti I.V. Triple arthrodesis: twenty-five and forty-four-year average follow-up of the same patients. J. Bone Joint Surg. Am., 1999, vol. 81, no. 10, pp. 1391-1402.

8. Segal L.S., Mann D.C., Feiwell E., Hoffer M.M. Equinovarus deformity in arthrogryposis and myelomeningocele: evaluation of primary talectomy. Foot Ankle, 1989, vol. 10, no. 1, pp. 12-16. DOI: 10.1177/107110078901000103

9. El-Mowafi H. Assessment of percutaneous V osteotomy of the calcaneus with Ilizarov application for correction of complex foot deformities. Acta Orthop Belg., 2004, vol. 70, no. 6. Pp. 586-590.

10. Ferreira R.C., Costo M.T., Frizzo G.G., da Fonseca Filho F.F. Correction of neglected clubfoot using the Ilizarov external fixator. Foot Ankle Int., 2006, vol. 27, no. 4, pp. 266-273. DOI: 10.1177/107110070602700407

11. Ferreira R.C., Costa M.T., Frizzo G.G., Santin R.A. Correction of severe recurrent clubfoot using a simplified setting of the Ilizarov device. Foot Ankle Int., 2007, vol. 28, no. 5, pp. 557-568. DOI: 10.3113/FAI.2007.0557

12. Kocaoglu M., Eralp L., Atalar A.C., Bilen F.E. Correction of complex foot deformities using the Ilizarov external fixator. J. Foot Ankle Surg., 2002, vol. 41, no. 1, pp. 30-39. DOI: 10.1016/s1067-2516(02)80007-2

13. Choi I.H., Kim J.I., Yoo W.J., Chung C.Y., Cho T.J. Ilizarov treatment for equinoplanovalgus foot deformity caused by melorheostosis. Clin. Orthop. Relat. Res., 2003, no. 414, pp. 238-241. DOI: 10.1097/01.blo.0000076801.53006.80 
14. Choi I.H., Yoo J.H., Chung C.Y., Cho T.J., Yoo W.J. Congenital diastasis of the inferior tibiofibular joint: report of three additional cases treated by the Ilizarov method and literature review. J. Pediatr. Orthop., 2004, vol. 24, no. 3, pp. 304-311. DOI: 10.1097/00004694-200405000-00012

15. Choi I.H., Yang M.S., Chung C.Y., Cho T.J., Sohn Y.J. The treatment of recurrent arthrogrypotic club foot in children by the Ilizarov method: A preliminary report. J. Bone Joint Surg. Br., 2001, vol. 83, no. 5, pp[. 731-737. DOI: 10.1302/0301-620x.83b5.11019

16. Dimeglio A., Bensahel H., Souchet P., Mazeau P., Bonnet F. Classification of clubfoot. J. Pediatr. Orthop. B, 1995, vol. 4, no. 2, pp. 129-136. DOI: $10.1097 / 01202412-199504020-00002$

17. Wainwright A.M., Auld T., Benson M.K., Theologis T.N. The classification of congenital talipes equinovarus. J. Bone Joint Surg. Br., 2002, vol. 84, no. 7, pp. 1020-1024. DOI: 10.1302/0301-620x.84b7.12909

18. Lee D.Y., Choi I.H., Yoo W.J., Lee S.J., Cho T.J. Application of the Ilizarov technique to the correction of neurologic equinocavovarus foot deformity. Clin. Orthop. Relat. Res., 2011, vol. 469, no. 3, pp. 860-867. DOI: 10.1007/s11999-010-1497-z

19. Kling T.F. Jr., Kaufer H., Hensinger R.N. Split posterior tibial-tendon transfers in children with cerebral spastic paralysis and equinovarus deformity. J. Bone Joint Surg. Am., 1985, vol. 67, no. 2, pp. 186-194.

20. Campbell W.C. An operation for the correction of "dropfoot". J. Bone Joint Surg., 1923, vol. 5, pp. 815.

21. Campbell W.C. Bone block operation for drop-foot. Analysis of end results. J. Bone Joint Surg., 1930, vol. 12, pp. 317-324.

22. Gill A.B. An operation to make a posterior bone block at the ankle to limit foot-drop. J. Bone Joint Surg., 1933 , vol. 15 , pp. 166.

23. Legaspi J., Li Y.H., Chow W., Leong J.C. Talectomy in patients with recurrent deformity in club foot. A long-term follow-up study. J. Bone Joint Surg. Br., 2001, vol. 83, no. 3, pp. 384-387. DOI: 10.1302/0301-620x.83b3.11051

24. Shalaby H., Hefny H. Correction of complex foot deformities using the V-osteotomy and the Ilizarov technique. Strategies Trauma Limb Reconstr., 2007, vol. 2, no. 1, pp. 21-30. DOI: 10.1007/s11751-007-0015-7

25. Carroll N.C. Controversies in the surgical management of clubfoot. Instr Course Lect., 1996, vol. 45, pp. $331-337$.

26. Carroll N.C., McMurtry R., Leete S.F. The pathoanatomy of congenital clubfoot. Orthop. Clin. North Am., 1978, vol. 9, no. 1, pp. 225-232.

27. Simons G.W. Complete subtalar release in club feet. Part I: A preliminary report. J. Bone Joint Surg. Am., 1985, vol. 67, no. 7, pp. 1044-1055.

28. Simons G.W. Complete subtalar release in club feet. Part II: Comparison with less extensive procedures. J. Bone Joint Surg. Am., 1985, vol. 67, no. 7 , pp. 1056-1065.

29. Viehweger E., Jacquemier M., Launay F., Giusiano B., Bollini G. First cuneiform osteotomy alters hindfoot architecture. Clin. Orthop. Relat. Res., 2005, vol. 441, pp. 356-365. DOI: 10.1097/01.blo.0000180605.535851.82

30. Atar D., Lehman W.B., Grant A.D. Complications in clubfoot surgery. Orthop Rev., 1991, vol. 20, no. 3, pp. $233-239$.

31. Franke J., Grill F., Hein G., Simon M. Correction of clubfoot relapse using Ilizarov's apparatus in children 8-15 years old. Arch. Orthop. Trauma Surg., 1990, vol. 110, no. 1, pp. 33-37. DOI: 10.1007/BF00431363

32. Grill F., Franke J. The Ilizarov distractor for the correction of relapsed or neglected clubfoot. J. Bone Joint Surg. Br., 1987, vol. 69, no. 4, pp. 593597. DOI: 10.1302/0301-620X.69B4.3611163

33. Dimeglio A., Bensahel H., Souchet P., Mazeau P., Bonnet F. Classification of clubfoot. J. Pediatr. Orthop. B, 1995, vol. 4, no. 2, pp. 129-136. DOI: 10.1097/01202412-199504020-00002

34. Wallander H., Hansson G., Tjernström B. Correction of persistent clubfoot deformities with the Ilizarov external fixator. Experience in 10 previously operated feet followed for 2-5 years. Acta Orthop. Scand., 1996, vol. 67, no. 3, pp. 283-287. DOI: 10.3109/17453679608994690

Received: 08.12.2020

\section{Information about the authors:}

1. Alexander P. Kirienko, M.D., Humanitas Clinical and Research Center - IRCCS, Rozzano, Milan, Italy, Email: alexander@kirienko.com

2. E. Malagoli, M.D.,

Humanitas Clinical and Research Center - IRCCS, Rozzano, Milan, Italy

3. G. Lucchesi, M.D.

Orthopaedic Clinic, Department of Surgery and Translational Medicine, University of Florence, Florence, Italy 